\title{
Von Bedarfen und Bedürfnissen - Gesundheit zwischen Solidarität und Kommerz
}

\author{
Karl-Ludwig Resch
}

Deutsches Institut für Gesundheitsforschung, Bad Elster, Deutschland

«Als Gesundheitsreform werden in Deutschland gesetzliche Eingriffe in die Rahmenbedingungen der Krankenversicherung bezeichnet. Diese Reformen dienten in den letzten Jahrzehnten meist der Stabilisierung des Beitragssatzes und waren in der Regel mit Einschränkungen der Leistungen, Erhöhung der Zuzahlungen an die sonst der Selbstverwaltung unterliegenden Versicherungen und Änderungen in der Bezahlung der Leistungserbringer verbunden.» So steht es bei Wikipedia $\mathrm{zu}$ lesen [1].

In der Tat, spätestens seit Mitte der 1970er Jahre Herbert Ehrenberg, damals Gesundheitsminister im Kabinett Helmut Schmidt, die «Kostendämpfung» als zentrale Philosophie für einen «gesetzlichen Eingriff in die Rahmenbedingungen der Krankenkassen» ausgegeben hat, treibt dieses Gedankengut im Gesundheitswesen sein Unwesen. Von explodierenden Gesundheitskosten ist da die Rede und von dramatisch steigenden Ausgaben. Nur durch rigorose Deckelung, Budgetierungen und andere dirigistische Maßnahmen scheint der große Kollaps immer wieder noch einmal soeben abwendbar. Sogar klassische Unwörter wie Rationierung oder Zweiklassenmedizin werden hie und da vernehmbar, wenngleich das Gesundheitsministerium erst kürzlich in einer Apologie des Gesundheitsfonds geradezu beschwörend Unmögliches beteuert: «So bleibt Spitzenmedizin für alle bezahlbar» [2].

Erst in allerjüngster Zeit scheint jemand bemerkt zu haben, dass die Gesetzliche Krankenversicherung (GKV) das Geld ihrer Mitglieder ja nicht aus dem Verkehr zieht und vernichtet, sondern in den Wirtschaftskreislauf einspeist. Also griff im Eckpunktepapier zur letzten Gesundheitsreform 2006 [3] die Erkenntnis Raum: «Deutschland hat ein modernes und leistungsfähiges Gesundheitswesen, das allen Bürgerinnen und Bürgern Zugang zu einer hochwertigen Gesundheitsversorgung und zugleich rund 4,2 Millionen Beschäftigten und Selbständigen Arbeitsplätze bietet. Das Gesundheitswesen ist damit eine dynamische Wirtschaftsbranche mit Innova- tionskraft und erheblicher ökonomischer Bedeutung für den Standort Deutschland.»

Die Einführung des sogenannten Gesundheitsfonds im Januar 2009 legt offen, welche Wirtschaftskraft mit der GKV assoziiert ist: etwa 150 Milliarden Euro pro Jahr. Und so macht allenthalben der Begriff der «Gesundheitswirtschaft» die Runde. Landesregierungen stricken an visionären Masterplänen oder outen ihren Beritt gar als «Gesundheitsland Nr. 1». Auf Bundesebene sponsort das Wirtschaftsministerium seit 2005 «Branchenkonferenz[en] Gesundheitswirtschaft» und prophezeit 2008 gar einen fundamentalen Paradigmenwechsel in diesem Wirtschaftssegment [4]. Ein eilends konstituierter ASTGeSo (Arbeitsstab Gesundheitswirtschaft und soziale Dienstleistungen) steht allen zur Seite, die die Tür zu diesem gigantischen Marktplatz nicht finden, an den die Erwartungen nicht hoch genug sein können. Stehen wir nach den Zeitaltern der Dampfmaschine, von Stahl und Eisenbahn, der elektrischen und chemischen Technik, des Automobils bzw. der Petrochemie sowie der Informationstechnologie am Beginn eines 6. Kondratieff-Zyklus, dem der Gesundheitswirtschaft? Beratungsgigant McKinsey prophezeit, dass «die Gesundheitsbranche mit einer Steigerung der Wertschöpfung um 3,3\% p.a. und einem Beschäftigungszuwachs um 1,9\% p.a. im Jahr 2020 eine der wichtigsten Säulen des Gesamtwachstums der deutschen Wirtschaft sein» kann [5]. Und Erzkonkurrent Roland Berger Consultants, die gleich zwei grundlegende Analysen in Serie anfertigten, können sich ein Wachstum von $70 \%$ bis 2020 auf dann gut 450 Milliarden Euro vorstellen [6] und sehen dabei eine zunehmende Marktorientierung: «Innovative Krankenkassen, Ärzte, Krankenhäuser oder Industrieunternehmen verlassen ihre angestammten Rollen und prägen den Gesundheitsmarkt mit unternehmerischem Denken» [7], wenngleich, wie betont wird, «der Nachfrage bislang noch kein ausreichendes Angebot gegenübersteht».

\section{KARGER}

Fax +497614520714

Information@Karger.de

www.karger.com (c) 2009 S. Karger GmbH, Freiburg

Accessible online at:

www.karger.com/fok
Prof. Dr. Karl-Ludwig Resch

Deutsches Institut für Gesundheitsforschung

Lindenstr. 5, 08645 Bad Elster, Deutschland

Tel. +49 3743755755 , Fax 55777

K.L.Resch@t-online.de 
Auf einer Konferenz zum Thema «Die Chancen der Gesundheitswirtschaft nutzen», zu der das Bundeswirtschaftsministerium Mitte 2008 einlud, wies einer der Referenten auf ein grundsätzliches, mit diesem Paradigmenwechsel einhergehendes Problem hin: «Ein ethisches Gut soll (wird) marktwirtschaftlich gesteuert (werden)». Offensichtlich ist sich die Politik der ökonomischen Potenziale weit mehr bewusst als der «Risiken und Nebenwirkungen» dieser Entwicklung. Immerhin ergab (m)ein Versuch, für die Problematik «Gesundheitlicher Verbraucherschutz» im Jahr 2008 einen Ansprechpartner $\mathrm{zu}$ finden, dass sich das Bundesgesundheitsministerium ebenso wenig für zuständig befand («das gehört zum Bereich Verbraucherschutz») wie das Bundesverbraucherschutzministerium («das gehört zum Bereich Gesundheit»). Da bleibt nur die marktwirtschaftliche Steuerung dieses ethischen Guts. Und marktwirtschaftlich heißt hier ganz klar: unter betriebswirtschaftlichen Gesichtspunkten. Also bekommt z.B. der chronisch kranke Mensch, der bislang «auf Krankenschein» einigermaßen gut medizinisch versorgt war, inzwischen viele Leistungen nur noch, wenn er sich als «schwerwiegend chronisch krank» qualifiziert. Alle anderen bleiben auf der Strecke, sprich sind einer unüberschaubaren Palette von Angeboten ausgesetzt, die primär von Marktfähigkeit und Rendite und nicht von gesundheitsfördernder oder Leiden lindernder Sinnhaftigkeit der Produkte und Dienstleistungen geprägt ist. Man mag zu der Perspektive, dass sich neben dem in seinem Volumen stagnierenden ersten ein dynamisch wachsender zweiter Gesundheitsmarkt entwickelt, stehen, wie man will - die Entwicklung scheint den Point of no Return längst überschritten zu haben. Roland Berger Consultants schätzen das Marktvolumen für 2006 bereits auf 60 Milliarden Euro [7].

Gerade deshalb ist es höchste Zeit, sich mit den grundlegenden Mechanismen stärker und professioneller zu beschäftigen, als dies bislang geschehen ist. Je mehr sich der zweite Gesundheitsmarkt entwickelt, umso unerlässlicher ist es, sich über die inhärenten Mechanismen des ersten wie des zweiten klar zu werden. Nicht nur, dass sich beide ergänzen, sie hängen auch untrennbar voneinander ab: Die Schwächen des ersten sind die Chancen des zweiten, die Schwächen des zweiten belasten den ersten.

Befasst man sich näher mit diesem Thema, fällt auf, dass kaum eine Quelle den Gesamtmarkt und seine Segmente durchdrungen hat. Immer wieder tauchen deutsche Begrifflichkeiten, wie z.B. Bedarf, Bedürfnis, Angebot oder Nachfrage, und englische, wie demand, needs, wants oder wishes, in unklarem Kontext auf. Eine Überprüfung dieser Begriffe im populären Internet-Wörterbuch Leo (http://dict.leo.org) ergab auch dort erstaunliche Unschärfen bei der Übersetzung von Begriffen wie Bedarf (demand, market, necessity, need, requirement, want), Bedürfnis (necessity, need, requirement, want), Nachfrage (demand, request, need, market), demand (Bedarf, Nachfrage, Verlangen, Begehren), want (Bedarf, Bedürfnis, Mangel).
Für die Wirtschaftswissenschaften steht, etwas vereinfacht gesagt, am Anfang das Bedürfnis. Daraus entsteht Nachfrage, und die definiert den Bedarf an Gütern oder Dienstleistungen, der auf dem Markt angeboten wird, um diese Bedürfnisse zu befriedigen. Folgerichtig entsteht aus dem Wunsch oder Bedürfnis (wish, want) nach einem neuen Auto eine Nachfrage (demand, request), die seitens des Autohändlers zu einem Bedarf mit entsprechenden Bestellungen beim Hersteller führt (market, need). Da viele Menschen ohne Auto weder zur Arbeit kommen würden, noch sich adäquat mit den Dingen des täglichen Lebens versorgen können, haben diese Menschen auch direkt einen objektiven Bedarf (need, necessity). - Auch der Wunsch bzw. das Bedürfnis nach zusätzlichen Klingeltönen fürs Handy erzeugt eine Nachfrage (demand, request), ohne dass dafür allerdings ein objektiver Bedarf (need, necessity) vorliegt - schließlich werden Handys serienmäßig mit einer akustischen Funktion ausgestattet, die ebenso zuverlässig etwaige Anrufer signalisiert wie jeder zusätzliche Klingelton. Bedarf und Bedürfnis sind also alles andere als obligate Synonyme, ganz besonders nicht im Gesundheitsmarkt.

Alle Deutschen, für die ja seit Kurzem eine explizite Versicherungspflicht besteht, haben einen gewissen «Anspruch» an Krankenkassen und Leistungserbringer. Diese haben nämlich nach $\S 70$ SGB V «eine bedarfsgerechte und gleichmäßige, dem allgemein anerkannten Stand der medizinischen Erkenntnisse entsprechende Versorgung der Versicherten zu gewährleisten. Die Versorgung der Versicherten muss ausreichend und zweckmäßig sein, darf das Maß des Notwendigen nicht überschreiten.» Damit werden gleichzeitig die Grenzen klar gezogen und der Begriff «Bedarf» auf die Attribute ausreichend und notwendig begrenzt. Was «Bedarf» ist, wird durch den Gesetzgeber, Expertenkommissionen und letztlich durch den Gemeinsamen Bundesausschuss definiert. Grundlage für den «Bedarf» an Rollstühlen z.B. sind Daten zur Inzidenz bzw. Prävalenz von Paraplegien, ergänzt um die Zweckmäßigkeit und Notwendigkeit dieses Bedarfs. Anders als in den Wirtschaftswissenschaften ist im Bereich der Gesundheitsversorgung bzw. des Gesundheitsmarktes also zu unterscheiden zwischen einem medizinisch begründeten Bedarf und einem eventuell deckungsgleichen, in der Regel aber deutlich darüber hinausgehenden Bedürfnis auf der Ebene des Einzelnen. Für zusätzliche Handyklingeltöne ließe sich nach diesem System sicher kein «Bedarf» ableiten. Gleichwohl besteht eine rege Nachfrage, hat sich ein beachtlicher Markt entwickelt. Auch für die Faltenunterspritzung mit Botox besteht medizinisch betrachtet kein Bedarf, gleichwohl scheint es vielen Menschen ein Bedürfnis zu sein (das sich in den USA inzwischen in über 3 Millionen Behandlungen pro Jahr abbildet). Ein Bedarf an Botox-Ampullen entsteht allenfalls für die «Leistungserbringer». Diese Differenzierung zwischen Bedarf und Bedürfnis ist systemimmanent und lässt sich auch bei «seriöseren» Produkten und Dienstleistungen beobachten. So wird zunehmend weniger ein sozialversicherungsrelevanter Bedarf zur Behandlung leichterer bis mittlerer Befindens- 
störungen oder unkomplizierter, selbstlimitierender Erkrankungen wie Schnupfen oder banale Erkältungen gesehen mit der Konsequenz, dass entsprechende Medikamente vom Schnupfenmittel bis zum Präparat gegen Regelschmerzen aus eigener Tasche zu bezahlen sind.

Die regelmäßigen Umfragen des Allensbach-Instituts zum Thema Naturheilmittel zeigen, dass inzwischen fast zwei Drittel der Bevölkerung grundsätzlich damit einverstanden sind, «die Verantwortung» selbst zu übernehmen, sprich selbst zu bezahlen, und dass es sich dabei um einen kontinuierlichen, einkommens- und bildungsunabhängigen Trend zur selbstbestimmten Nutzung von Naturheilmitteln mit einer Zustimmungsquote von fast $75 \%$ in der Bevölkerung handelt [8]. Dies entspricht durchaus dem, was Trendforscher schon seit Jahren vorhersagen, z.B. «dass neben allen wirtschaftlichen und finanziellen Notwendigkeiten, die den Megatrend Gesundheit unterstützen, der gesunde Nachfragemarkt auch durch die wachsende Lust der Menschen an der Beschäftigung mit dem eigenen Wohlbefinden katalysiert» wird [9].

Neben der wachsenden Lust darf auch der wachsende Frust als Triebfeder für eigenständige Initiativen nicht unterschätzt werden. «Mehr als 50\% aller Patienten in der Praxis des Allgemeinarztes klagen über allfällige Beschwerden ohne klaren körperlichen Befund und werden oft teuren und gefährlichen diagnostischen Prozeduren ausgesetzt, die weder eine angemessene wissenschaftliche Grundlage noch therapeutische Konsequenz haben», wurde vor einigen Jahren in einem Artikel der Süddeutschen Zeitung konstatiert [10]. Wer sich in der medizinischen Fachliteratur auf die Suche macht, findet überraschend viele Artikel, die sich exakt mit dieser Problematik beschäftigen und das Zitat grundsätzlich bestätigen. Top-Journale wie das British Medical Journal nehmen sich in den letzten Jahren zunehmend der Thematik «medically unexplained symptoms» an [z.B. 11,12], für die gerade naturheilkundliche, komplementäre und alternativmedizinische Ansätze von besonderer Bedeutung sind und die deshalb auch in ForsCHENDE KOMPLEMENTÄRMEDIZIN immer wieder thematisiert werden [13-15]. In einer von mehreren Publikationen einer Serie zu diesem Thema in den Annals of Internal Medicine stellen die Autoren fest, dass man hier «viel von den Ärzten der Prä-Freud-Ära lernen» könne [16], ... in der die tragenden Säulen Konzepte und Strategien waren, die heute die sogenannte CAM-Szene prägen, könnte man ergänzen. Und die in der Lage sind, losgelöst von einem offiziell festgelegten Bedarf unbefriedigte Bedürfnisse nach mehr Wohlbefinden sensibel zu bedienen. Vielleicht ließe sich damit sogar die lange, erbitterte Diskussion um die Priorität von hoher «perceived effectiveness» oder methodisch exzellenter «efficacy» mit salomonischer Zweigleisigkeit entzerren.

Fazit: Die Differenzierung zwischen einem sozialversicherungsgeprägten, paternalistisch vorgegebenen «Bedarf» und einem darüber weit hinausgehenden, gegebenenfalls auch davon unabhängigen, vom Einzelnen definierten «Bedürfnis» ist der Schlüssel zu einer wirtschaftlich erfolgreichen Entwicklung des zweiten Gesundheitsmarkts, in dem gerade für die therapeutischen Angebote eine hohe Nachfrage zu erwarten ist, welche die individuellen Vorstellungen und Wünsche, Möglichkeiten und Grenzen vieler Patienten adäquat bedienen. Eine überaus gute Perspektive für eine ganze Reihe von Verfahren aus der Welt der CAM. Und eine Herausforderung für alle Anbieter, die die Grenzen dessen überschreiten, was aus Sicht der Sozialversicherung notwendig ist. Sie müssen sich auf das Sinnvolle beschränken und dürfen sich nicht auf Unsinniges oder gar Gefährliches einlassen. Darin liegen die eigentliche Herausforderung und die größte Gefahr für die nachhaltige Entwicklung des zweiten Gesundheitsmarkts.

\section{Literatur}

1 http://de.wikipedia.org/wiki/Gesundheitsreform (letzter Zugriff: 01.02.2009).

2 Magazin für Soziales, Familie und Bildung 11/2008, Nr. 069. Presse- und Informationsamt der Bundesregierung www.bundesregierung.de/Content/DE/ Magazine/MagazinSozialesFamilieBildung/069/anlagen/PDF-Version_20zum_20Ausdrucken,proper ty=publicationFile.pdf.

3 Eckpunkte zu einer Gesundheitsreform 2006. CDU/CSU-Bundestagsfraktion. www.cdu.de/doc/ pdfc/060704_eckpunkte_gesundheit.pdf.

4 Von der Gesundheitsversorgung zur Gesundheitswirtschaft - ein Paradigmenwechsel. Schlaglichter der Wirtschaftspolitik. Monatsbericht September 2008. Bundesministerium für Wirtschaft und Technologie (BMWi). http://lexikon.bmwi.de/BMWi/Navigation/Medienraum/infografiken, did=268298.html.

5 Deutschland 2020. Zukunftsperspektiven für die deutsche Wirtschaft. Frankfurt, McKinsey und Company, 2008. www.mckinsey.de/html/profil/initiativen/d2020.asp.
6 Kartte J, Neumann K, Kainzinger F, Henke KD: Innovation und Wachstum im Gesundheitswesen. Roland Berger Strategy Consultants, 2005. www.rolandberger.com/media/pdf/rb_press/RB_study_Innovation_and_growth_healthcare_D_20051102.pdf.

7 Kartte J, Neumann K: Der Zweite Gesundheitsmarkt. Die Kunden verstehen, Geschäftschancen nutzen. Roland Berger Strategy Consultants, 2007. Kostenlose Hardcopy erhältlich unter: www.rolandberger.com/services/form/com_rb_3704106666.

8 Naturheilmittel 2002. Institut für Demoskopie Allensbach, 2002. www.ifd-allensbach.de/pdf/Akt_ 0201.pdf.

9 Mühlhausen C: Megamarkt Gesundheit. Die Schlüsselbranche der Zukunft - 14 Trends für das Health-Business von morgen. Kelkheim, Zukunftsinstitut 2003. www.zukunftsinstitut.de/verlag/zukunftsdatenbank_suche.php?nr=976.

10 De Ridder M, Dissmann W: Notfall Medizin. Süddeutsche Zeitung, 27 Mai 2000.
Rosendal M, Olesen F, Fink P: Management of medically unexplained symptoms. BMJ 2005;330:4-5.

12 Hatcher S, Arroll B: Assessment and management of medically unexplained symptoms. BMJ 2008;336: 1124-8.

13 Esch T, Stefano GB: A bio-psycho-socio-molecular approach to pain and stress management. Forsch Komplementmed 2007;14:224-34.

14 Stange R, Pflugbeil C: Fasten im Rahmen einer naturheilkundlichen Komplextherapie der Polymyalgia rheumatica. Forsch Komplementmed 2007;14: 235-9.

15 Musial F, Michalsen A, Dobos G: Functional chronic pain syndromes and naturopathic treatments: neurobiological foundations. Forsch Komplementmed 2008;15:97-103.

16 Sharpe M, Carson A: «Unexplained» somatic symptoms, functional syndromes, and somatization: do we need a paradigm shift? Ann Intern Med 2001; 134:926-30. 